\title{
Discussion avec Fabian Goncalves Borrega sur la photographie au Musée d'art des Amériques
}

Interview with Fabian Goncalves Borrega about photography at the Art Museum of the Americas

Entrevista con Fabian Goncalves Borrega sobre la fotografía en el Museo de arte de las Américas

\section{Laura Cahier}

\section{OpenEdition}

\section{Journals}

Édition électronique

URL : http://journals.openedition.org/ideas/4826

DOI : $10.4000 /$ ideas.4826

ISSN : $1950-5701$

Éditeur

Institut des Amériques

\section{Référence électronique}

Laura Cahier, «Discussion avec Fabian Goncalves Borrega sur la photographie au Musée d'art des Amériques », IdeAs [En ligne], 13 | 2019, mis en ligne le 01 mars 2019, consulté le 21 avril 2019. URL http://journals.openedition.org/ideas/4826 ; DOI : 10.4000/ideas.4826

Ce document a été généré automatiquement le 21 avril 2019

\section{c) (7) $९$}

IdeAs - Idées d'Amériques est mis à disposition selon les termes de la licence Creative Commons Attribution - Pas d'Utilisation Commerciale - Pas de Modification 4.0 International. 


\section{Discussion avec Fabian Goncalves Borrega sur la photographie au Musée d'art des Amériques}

Interview with Fabian Goncalves Borrega about photography at the Art Museum of the Americas

Entrevista con Fabian Goncalves Borrega sobre la fotografía en el Museo de arte de las Américas

Laura Cahier

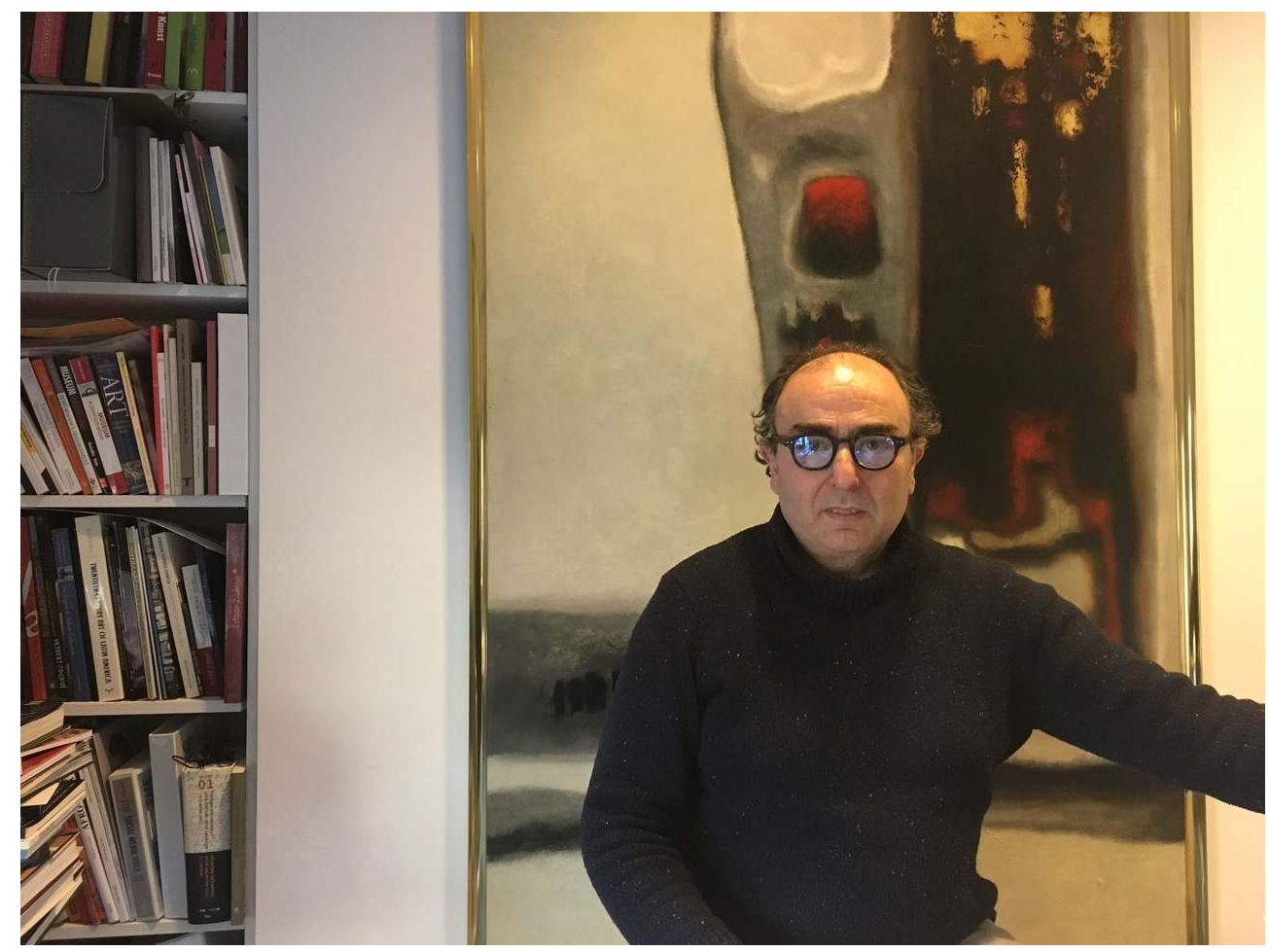

(c) Laura Cahier, 2019. 
1 La discussion que nous avons eue avec Fabian Goncalves Borrega, commissaire d'expositions et conservateur de la photographie au Musée d'art des Amériques (Art Museum of the Americas, AMA), est née d'une volonté de comprendre la portée et l'influence de la photographie engagée dans les Amériques ainsi que le rôle de l'AMA dans la promotion de cette forme de représentation et d'expression artistique.

2 Situé au cœur de la ville de Washington, l'AMA est l'un des plus anciens musées d'art moderne et contemporain des États-Unis. Héritier du Visual Arts Unit de l'Union panaméricaine, il est aujourd'hui intégré à l'Organisation des États américains (OÉA), dont le but est de promouvoir la démocratie, la justice, la paix et la solidarité parmi ses trente-cinq États membres. De la nouvelle figuration à l'abstractionnisme géométrique, en passant par l'art conceptuel, cinétique ou optique, la collection de l'AMA compte plus de deux-cents œuvres, toutes issues des différents courants artistiques qui ont traversé l'Amérique latine et les Caraibes depuis le $\mathrm{xx}^{\mathrm{e}}$ siècle. En 2005, un second espace d'expositions, la Photogalerie, a été ouvert au sein même de l'OÉA et à quelques pas seulement du bâtiment historique du musée.

\section{Musée d'art des Amériques}

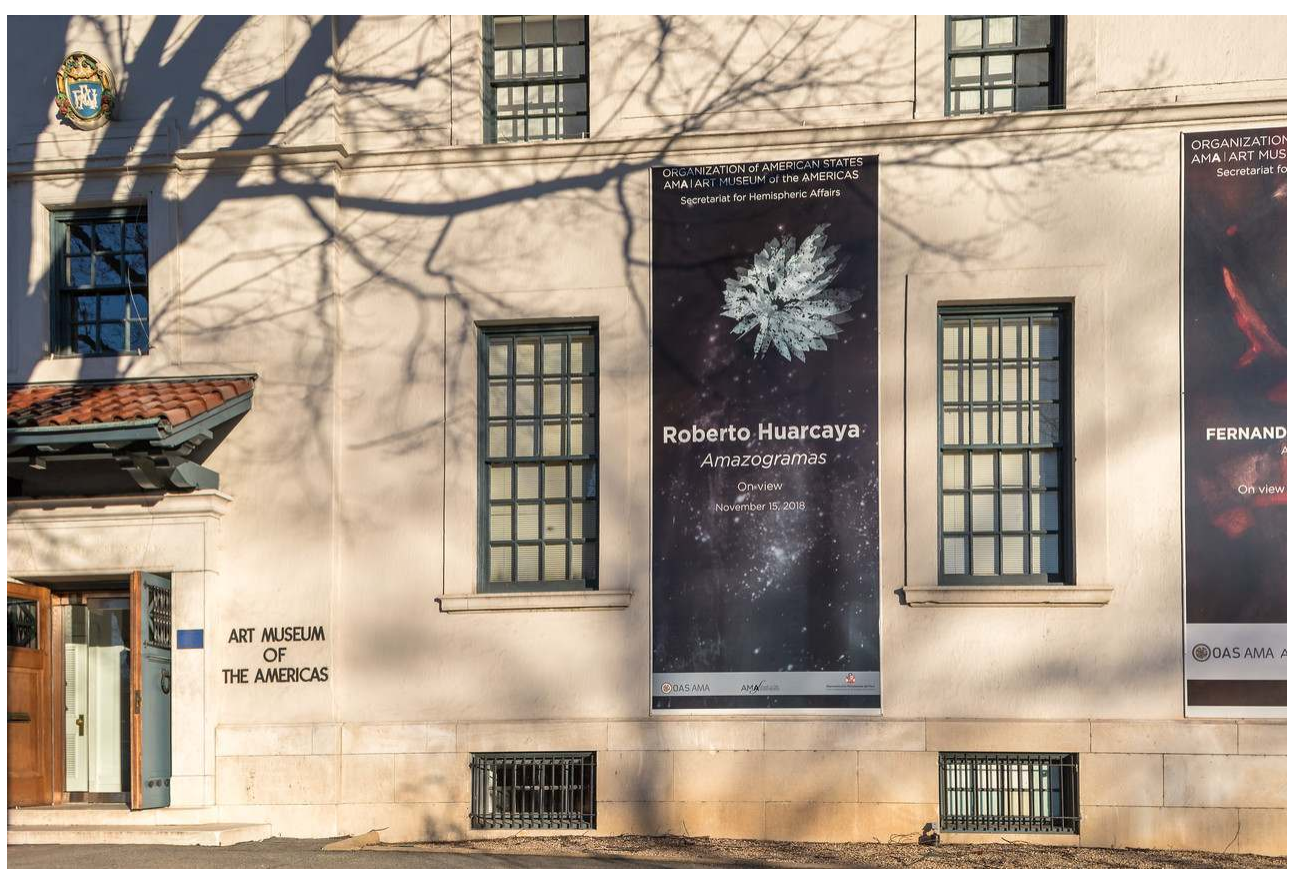

(c) Rafa Cruz, 2018 (reproduit avec l'aimable autorisation du photographe)

LAURA CAHIER : En guise d'introduction à cet échange, pourriez-vous nous présenter votre parcours professionnel et ce qui vous a amené jusqu'au Musée d'art des Amériques (AMA)?

Fabian Goncalves Borrega : Initialement, j'ai une formation de conservateur de musée et, plus particulièrement, de restaurateur de mobilier ancien. J'ai commencé à travailler en 1986 à Buenos Aires dans le Musée d'art hispano-américain Isaac Fernández Blanco, qui rassemble la collection la plus importante de meubles hispano-américains au monde. Ce fut, en quelque sorte, mon premier pas dans le monde des musées. En tant que conservateur de musée, j'ai alors été amené à prendre de nombreuses photos afin d'avoir des images des objets et meubles à préserver. Je me suis donc rapidement intéressé à la photographie. Et puis mon père était un photographe amateur: il m'a 
appris toutes les techniques nécessaires afin que l'appareil photo réponde à l'intention du photographe et non l'inverse. Il faut dire qu'à cette époque les appareils photos étaient bien moins faciles d'utilisation qu'ils ne le sont aujourd'hui. De cette manière, mon intérêt pour les musées d'un côté et ma passion pour la photographie de l'autre se sont mêlés et complétés.

En 1992, j'ai obtenu un visa américain grâce à la Green Card Lottery et j'ai pu me rendre aux États-Unis, où j'ai trouvé un poste de consultant. J'ai d'abord travaillé au sein de la Smithsonian Institution, notamment au Musée national d'histoire américaine. En 1998, j'ai obtenu un poste de commissaire d'expositions temporaires à l'AMA. À ce moment de ma carrière, j'étais vraiment porté sur la question de la conservation des œuvres d'art, puisque la seule manière de ne pas avoir à restaurer une pièce est d'éviter que celle-ci ne s'abîme. Mes collègues de l'AMA et moi-même avions donc le défi de préserver toutes les collections détenues par le musée, soit plus de deux-cents œuvres!

À partir de ce moment-là, j'ai rapidement lancé l'idée d'une exposition photographique dans la galerie du musée. Nous sommes parvenus à faire voir le jour à ce projet, qui était alors inédit! En 1998, Luis Delgado, un photographe mexicain vivant maintenant à San Francisco, a exposé ses travaux. Nous avons alors décidé de continuer sur cette lancée et de promouvoir davantage la photographie. En 2005, nous avons ouvert la Photogalerie, qui se situe dans le bâtiment même de l'OÉA, au niveau de la $F$ Street. Nous avons pris cette décision dans un contexte où le monde de la photographie était en pleine explosion. Grâce aux téléphones portables et aux appareils numériques, la photographie connaît une démocratisation inédite. Les ordinateurs peuvent recevoir, enregistrer et renvoyer des images. Les photos ont pris une place prépondérante dans cette «nouvelle vie numérique ", et ce également grâce à des plateformes de partage telles que Facebook ou MySpace. Progressivement, le niveau de culture photographique s'est amélioré et nous sommes tous devenus des critiques photographiques. Sur Facebook, le "pouce levé » est le symbole de cette critique, puisqu'il permet à tout individu de dire s'il aime ou non telle ou telle photo. La photographie est devenue, en quelques années, un media socialement accepté. Elle s'est même hissée au cœur des préoccupations de nombreux individus qui, désormais, font particulièrement attention à leurs photos de "profil» et soignent les photos qu'ils publient. Dans ce contexte, avec mes collègues, nous nous sommes toutefois dit que ces photographies et ces images n'avaient pas d'existence tangible, mais seulement numérique. Et puis, elles ne proposent qu'un seul niveau d'interprétation, souvent manifeste et explicite. En fait, la plupart de ces photos ne sont pas des photographies artistiques.

Lorsque nous avons ouvert la Photogalerie, en 2005, nous avons commencé à travailler avec la collection de photographies que l'AMA détenait déjà. Nous avons dû nous atteler à une double tâche : d'une part, étudier et comprendre le contenu et le sens de la collection photographique afin de l'organiser en thèmes distincts ; d'autre part, faire le point sur les pièces qu'il nous manquait afin de pouvoir compléter la collection par de nouvelles acquisitions. J'ai alors commencé à consulter des portfolios de photographes et à me rendre dans des lieux où il y avait une offre photographique conséquente. Je suis d'abord allé à Fotofest International, à Houston dans l'État du Texas. Puis, petit à petit, nous avons été invités à d'autres festivals, comme le Festival de la Luz à Buenos Aires, le FestFoto de Porto Alegre, Lima Photo au Pérou, ainsi qu'à New York ou encore à Portland dans l'État de l'Oregon.

Voilà, en quelques mots, le parcours qui m'a amené jusqu'à mon poste actuel de commissaire d'expositions et de conservateur de la photographie pour l'AMA. 
Actuellement, je suis chargé de toute la programmation et de la mise en place concrète des expositions du musée ainsi que de la Photogalerie.

L.c. : Je vous remercie d'être revenu sur le riche parcours qui vous a conduit jusqu'à l'AMA Cela m'amène à vous questionner sur le rôle de l'Organisation des États américains (OÉA) en matière de promotion de la photographie et, plus généralement, de l'art. Est-ce que l'OÉA a développé une politique culturelle spécifique à cet égard?

F.G.B. : L'OÉA a une mission qui repose sur quatre piliers : la démocratie, la sécurité, la liberté d'expression et le développement durable. Au sein de l'onglet "démocratie ", nous trouvons l'idée très importante, à mon avis, de représentation et de droits des minorités vivant dans les Amériques. Dans ce contexte, l'AMA répond, je crois, à un objectif de soft diplomacy. À travers l'exposition d'œuvres artistiques, la fonction de l'AMA est de donner à voir certaines problématiques aux visiteurs afin qu'émerge une prise de conscience, tout en cherchant des formes de représentation inclusive aptes à susciter et renouveler le dialogue. En ce sens, l'AMA s'aligne complètement avec les objectifs de l'OÉA qui sont édictés par le secrétaire général de notre organisation. Personnellement, en tant que coordinateur d'expositions, j'ai vraiment cherché à m'intéresser à la question de la représentation et de la démocratie, plus qu'à la thématique de la sécurité par exemple.

L.c. : Comment s'effectue le choix des artistes dont les travaux seront exposés à l'AMA et à la Photogalerie?

F.G.B. : Le choix des artistes se fait différemment selon qu'il s'agisse du Musée ou de la Photogalerie. Concernant le Musée, nous avons un panel de cinq conservateurs issus de la Smithsonian Institution et d'autres organisations internationales ayant des programmes culturels, telles que la Banque mondiale ou la Banque internationale pour la reconstruction et le développement. Nous leur soumettons les candidatures des artistes pour le Musée et ils les approuvent ou non. Concernant la Photogalerie, je suis moi-même chargé du visionnage des portfolios et de la sélection des photographes. Dans un second temps, je soumets mes trouvailles à un comité de conservateurs de musée issus de l'AMA et nous décidons, ensemble, des prochains photographes dont les travaux seront exposés.

L.c. : Prenez-vous en compte les «quatre piliers» de l'OÉA dans la sélection des photographes?

F.G.B. : En fait, je pense avant tout les expositions comme des histoires se rapportant à des personnes, à des objets ou à des thèmes. Je dirais que les trois critères qui importent lorsque je choisis un photographe sont à la fois cette capacité à raconter une histoire, la qualité intrinsèquement artistique des images et, enfin, le lien avec les quatre piliers de l'OÉA. Les images doivent dire quelque chose, elles doivent avoir plus qu'un seul niveau d'interprétation: c'est cela qui donne de la complexité à la photographie elle-même et, in fine, à l'histoire.

L.c. : Est-ce qu'il s'agit seulement de photographes originaires des Amériques ?

F.G.B.: Les photographes doivent avoir une connexion avec l'Amérique latine, les Caraïbes ou l'Amérique du Nord. Évidemment, nous sommes limités dans les expositions que nous pouvons présenter, si bien qu'il nous faut un lien avec le continent, que ce soit l'origine du photographe ou le lieu où les photos ont été prises. Par ailleurs, l'OÉA a aussi des pays observateurs, tels que la France, l'Italie et l'Espagne, de qui nous pouvons aussi collecter des photos. Globalement, j'ai remarqué que de nombreux photographes latino-américains travaillent aujourd'hui aux États-Unis. Je ne 
sais pas si c'est une tendance qui va perdurer, mais il s'agit d'un nombre conséquent d'artistes qui viennent ici pour trouver un soutien financier et institutionnel.

L.c. : Croyez-vous qu'il soit plus facile de promouvoir la photographie, comme œuvre d'art, en Amérique du Nord qu'en Amérique latine?

F.G.B.: En fait, je pense que l'Amérique du Nord offre davantage d'opportunités culturelles en termes d'expositions et de festivals, ainsi que de meilleures conditions matérielles et financières. Par exemple, tous les outils et matériaux qu'utilisent les photographes sont moins chers ici, puisqu'ils n'ont pas besoin d'être importés, comme c'est le cas en Amérique latine. Par ailleurs, il est plus facile d'avoir un écho dans le monde de la culture, en étant dans des villes comme New York, Washington ou San Francisco. "Nul n'est prophète en son pays »: c'est là notre problème, il faut suivre notre destin et aller là où se trouvent les meilleures opportunités. En plus, il était jusqu'à présent assez facile de se faire accepter aux États-Unis. Mais les chosent sont actuellement en train de changer...

L.c. : Fabian, la première fois que nous nous sommes rencontrés, vous m'aviez présenté deux expositions photographiques. La première d'entre elles, exposée au premier étage de I'AMA, donnait à voir le travail du photographe péruvien Roberto Huarcaya. Pourriez-vous nous en dire un peu plus, sur l'œuvre de cet artiste?

F.G.B. : Roberto Huarcaya est un bon exemple du travail que nous faisons avec l'AMA et, d'ailleurs, sa candidature a tout de suite été acceptée par le comité de sélection. Pourquoi ? Parce que l'Amazonie, que Roberto Huarcaya capture dans ses photos, est le poumon de notre planète. Cependant, elle est aujourd'hui menacée par les activités humaines. Le projet artistique de Roberto Huarcaya s'intitule Amazogramas : il s'agit d'une série de photogrammes réalisés dans le parc national de Bahuaja Sonene, dans la forêt péruvienne de Tambopata. Grâce à la technique qu'il utilise, à savoir le photogramme, il démontre que l'on peut produire quelque chose à partir de la forêt amazonienne sans la détruire. Au travers du processus même de production artistique, il appelle à une prise de conscience du public au sujet de la préservation de la forêt. En fait, cela correspond exactement à l'objectif de développement durable de l'OÉA. Et puis, son projet est fascinant et artistiquement réussi ! Je crois, d'ailleurs, qu'il y a eu aussi une certaine prise de conscience de la part des autorités nationales elles-mêmes, car Roberto Huarcaya a eu besoin de l'autorisation du parc national de Bahuaja Sonene afin de pouvoir réaliser ces photogrammes. Si vous voulez, pour pouvoir prendre une telle photo, il faut une conjonction d'événements positifs et celle-ci peut être compromise par les nombreux problèmes qu'il y a dans la région, tels que les trafics de drogues, la déforestation illégale ou encore les passeurs. 
Le projet Amazogramas de Roberto Huarcaya

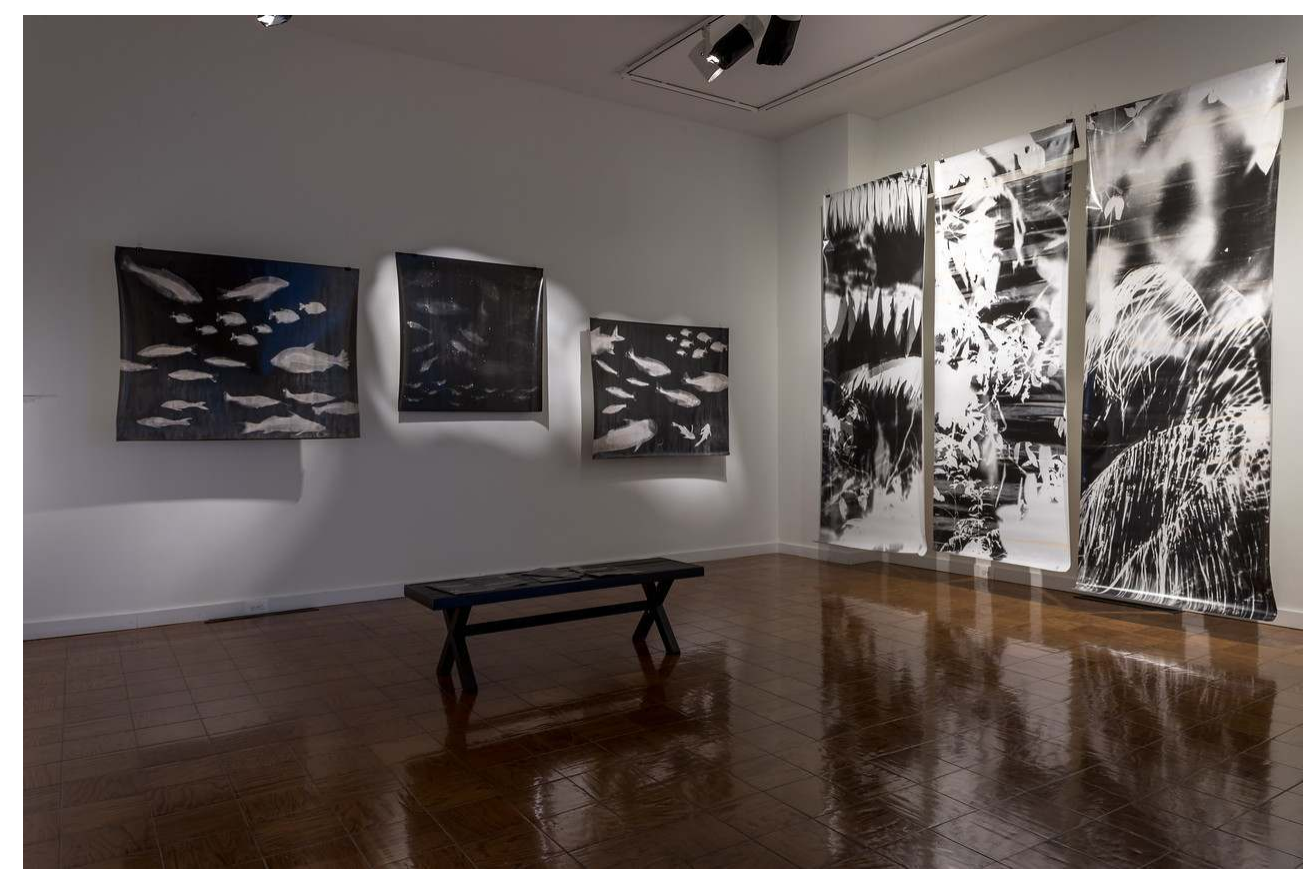

(c) Rafa Cruz, 2018 (reproduit avec l'aimable autorisation du photographe).

L.c. : Pourriez-vous nous parler de la technique que Roberto Huarcaya utilise pour réaliser ces photogrammes, sans justement détruire la forêt amazonienne?

F.G.B. : En fait, Roberto Huarcaya peint avec la lumière ! Dans un premier temps, il s'est rendu dans la forêt de Tambopata afin de repérer l'endroit qu'il souhaitait photographier. Une fois le repérage effectué et les autorisations obtenues, il a placé une bande de papier photosensible mesurant 30 mètres de long par 1,5 mètre de large dans la forêt, durant la nuit. Il a positionné cette bande entre les arbres et les feuillages, en évitant ainsi d'abîmer l'environnement. Ensuite, il a utilisé une lampe torche, c'est-àdire une source de lumière, pour illuminer les éléments de la forêt. Quand la lumière est obstruée par une feuille ou des insectes, apparaissent alors des zones plus ou moins grises sur le papier photosensible. En fait, tout au long de ce processus, il n'utilise aucun appareil photo. Je vous le disais, Roberto peint avec de la lumière ! Ce qui est intéressant, c'est que l'accent est vraiment mis sur le processus de création artistique : le résultat final, c'est-à-dire la bande de papier photosensible qui est exposée, laisse apparaître des accidents et des erreurs, comme la pluie ou les rayons lumineux de la lune.

L'exposition Amazogramas s'intègre dans un projet plus large, qui donne à voir de manière originale et spéciale les trois éléments qui définissent le Pérou : la forêt, la montagne et la mer. Et, de façon évidente, ces trois thèmes sont liés au développement durable. On pourrait résumer le travail de Roberto de la façon suivante : entrer dans une zone, faire son travail et repartir sans avoir modifié ou abîmé l'environnement. Par exemple, tous les produits chimiques qui ont été utilisés pour réaliser le photogramme ont été importés depuis Lima, puis ramenés dans la capitale afin d'y être recyclés. 
Une partie de la bande photosensible de 30 mètres de long composant le projet Amazogramas de Roberto Huarcaya

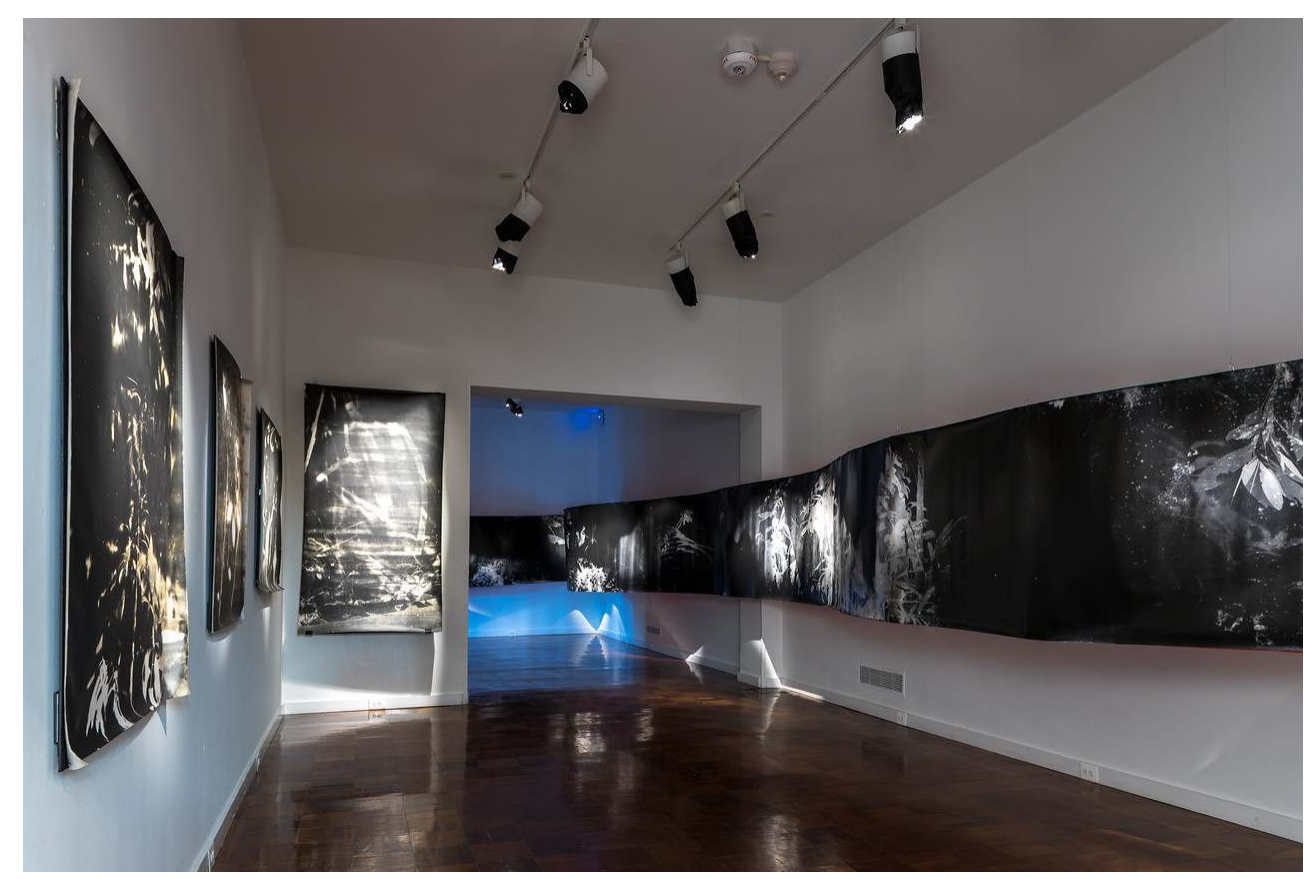

(c) Rafa Cruz, 2018 (reproduit avec l'aimable autorisation du photographe).

L.c. : A-t-il travaillé avec des communautés locales pour réaliser ces photogrammes ?

F.G.B. : Oui, tout à fait. Il lui a fallu trois ans avant de pouvoir commencer à réaliser les photogrammes. Durant ces trois années, il est revenu plusieurs fois dans la zone, ce qui a intrigué les locaux. En fait, les gens étaient inquiets de ce qu'il venait faire dans la forêt, car il y a énormément de problèmes d'exploitation des ressources et de trafics dans cette zone. Petit à petit, les habitants lui ont posé des questions sur ce qu'il faisait sur leurs terres. Roberto est ainsi entré en contact avec les populations locales et il a d'ailleurs embauché des habitants afin de l'aider dans ce projet.

L.c. : La seconde exposition présentée dans la Photogalerie donne à voir l'œuvre du photographe Roberto Fernández Ibáñez et fait d'ailleurs écho au travail de Roberto Huarcaya. Pourriez-vous nous parler un peu plus de cette exposition? 


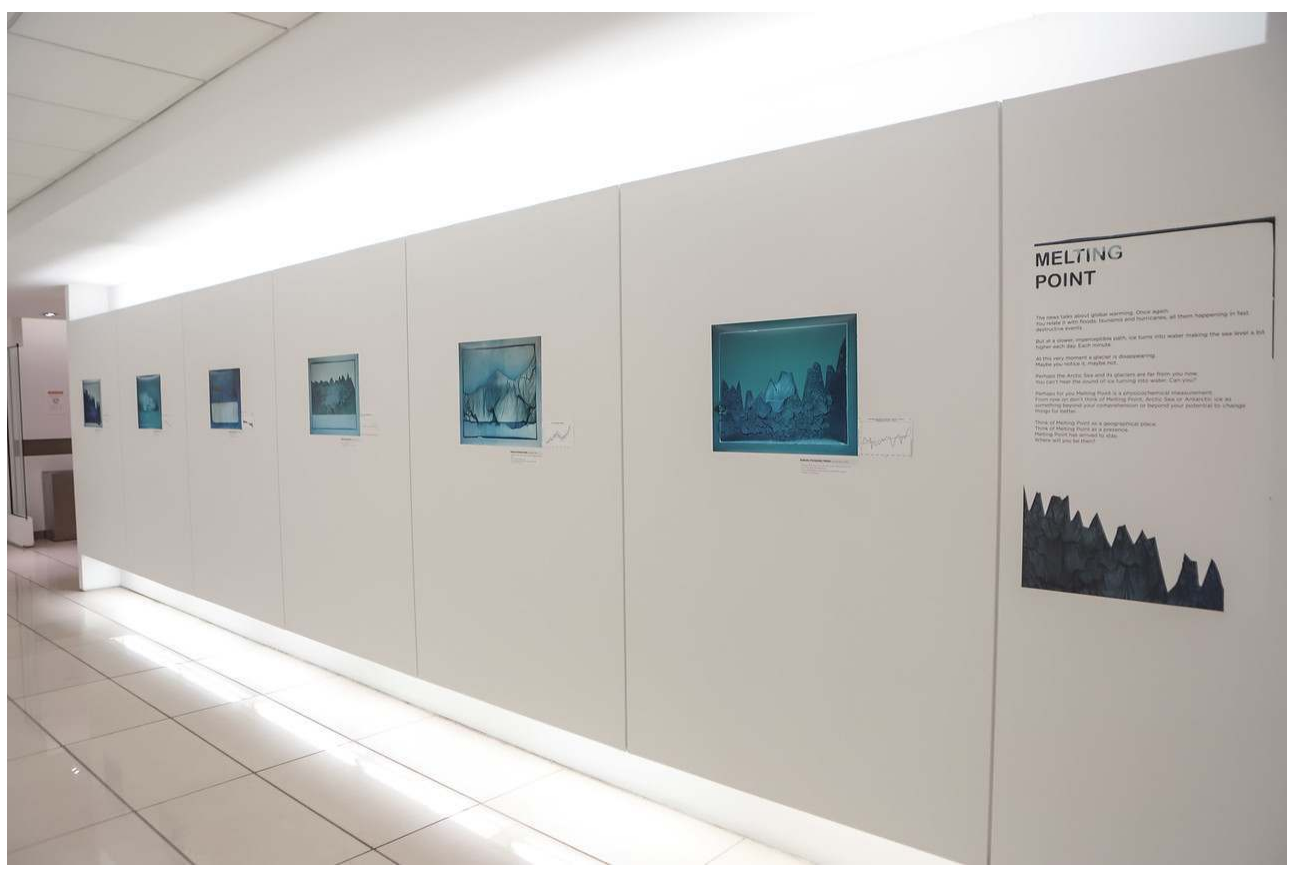

(c) Rafa Cruz, 2018 (reproduit avec l'aimable autorisation du photographe).

F.G.B.: Roberto Fernández Ibáñez est un photographe uruguayen, qui cherche constamment à élargir la manière dont nous percevons la planète Terre. Il a réalisé quatre séries de photogrammes : Earthy Resilience, Melting Point, Rara Avis et The Hand. Le projet Melting Point reprend les inquiétudes que nous avons, dans le Sud du continent, concernant la fonte des glaces en Antarctique. Il utilise une technique formidable et très ancienne, celle du cyanotype. Dans un premier temps, il met au point un mélange photosensible à partir de ferricyanure de potassium et de citrate d'ammonium, qu'il applique ensuite sur une feuille de papier. Une réaction chimique se produit et permet d'obtenir différentes nuances de bleu. Et ce bleu est magnifique, si profond, si pur ! En fait, il utilise ces teintes pour parler de la fonte des glaces, car le bleu qu'il obtient rappelle vraiment la couleur de la glace. Dans un second temps, il utilise ces images pour reproduire des graphiques relatifs à l'évolution de la quantité de glace selon les saisons. Ce sont principalement des graphiques publiés par la NASA et qui retracent l'évolution des quantités de glace en Antarctique. Enfin, avec ce projet, il s'intéresse aux effets du réchauffement climatique... 


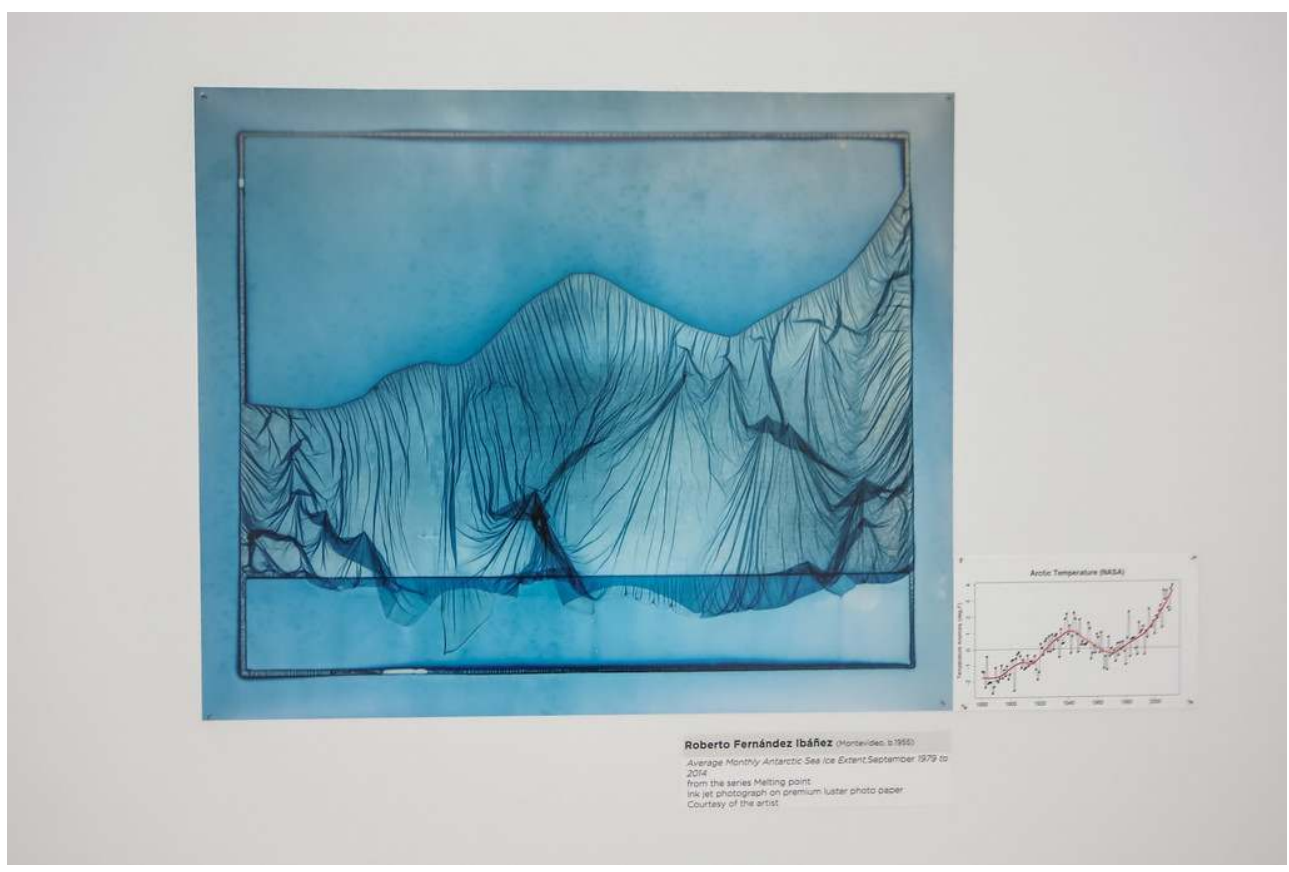

(c) Rafa Cruz, 2018 (reproduit avec l'aimable autorisation du photographe).

Avec le projet Rara Avis, Roberto Fernández Ibáñez propose une analogie entre le dernier vol de la feuille de l'arbre vers le sol et la présence ou l'absence de vie. Pour ce faire, il recompose une scène avec des éléments naturels, qu'il photographie ensuite. Puis, il travaille individuellement chaque négatif, en faisant réagir le papier avec des produits chimiques variés, dans le but d'obtenir des couleurs différentes. Au-delà d'une opération chimique, il intervient aussi mécaniquement en utilisant du papier de verre pour changer la texture du négatif. Ce projet est une pure création, il fait preuve d'une liberté totale par rapport aux normes photographiques, et ce afin d'attirer l'attention sur la disparition des espèces naturelles et de la biodiversité. En fait, l'idée est de montrer que, quand toutes les espèces naturelles auront péri, il nous restera seulement des photos prises dans le passé. C'est une manière artistique et visuellement intéressante d'attirer l'attention sur une problématique liée au développement durable.

Concernant le projet Earthy Resilience, il donne à voir différentes représentations de la planète Terre, en les agrémentant de retouches d'or, selon la technique japonaise du kintsugi ${ }^{1}$. Cela permet de donner du caractère à la photographie, tout en montrant la résilience de notre planète, la Terre. La métaphore à retrouver derrière ce projet est la suivante : la Terre est en train de souffrir du fait des activités humaines et des cicatrices apparaissent un peu partout sur le globe. Mais, en recouvrant ces marques douloureuses et profondes avec des pigments d'or, Roberto Fernández Ibáñez valorise la résilience de notre planète. 
Projet Earthy Resilience de Roberto Fernández Ibáñez

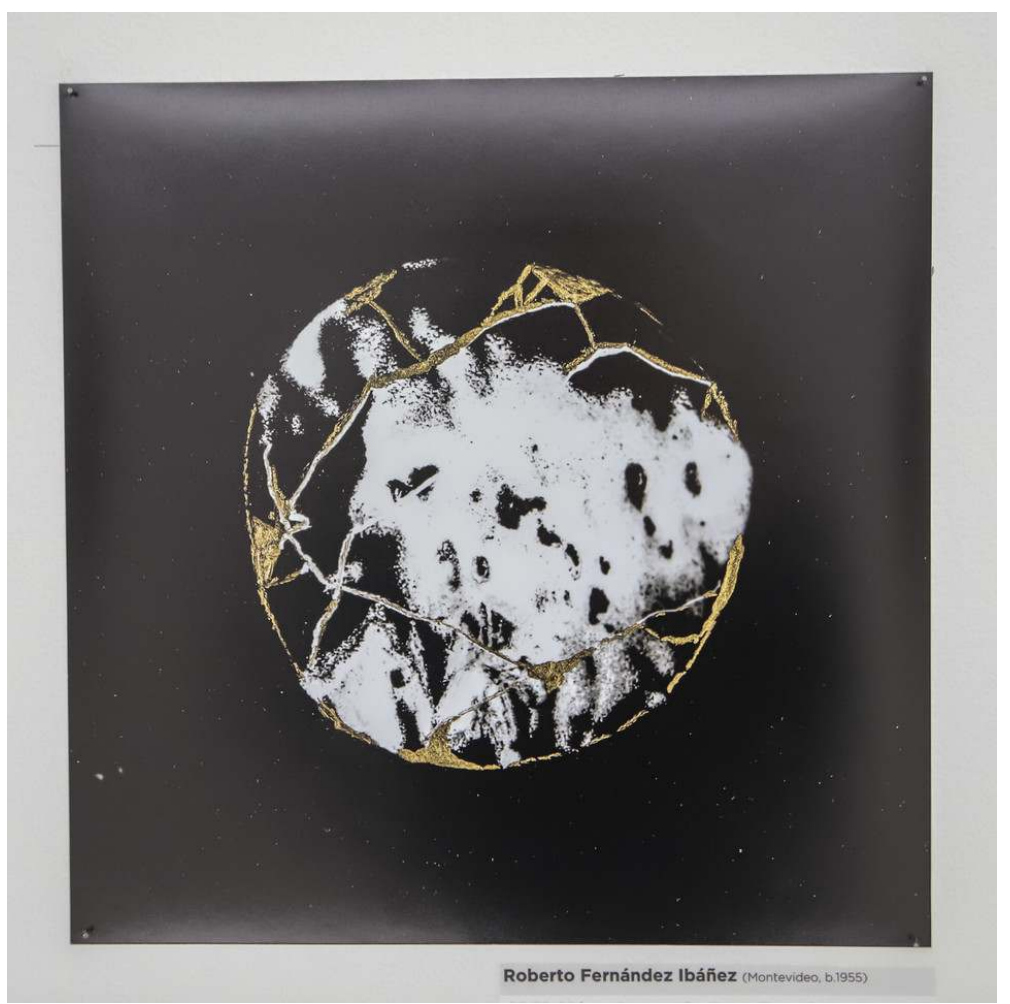

(c) Rafa Cruz, 2018 (reproduit avec l'aimable autorisation du photographe).

Enfin, le projet The Hand est né d'une expérience personnelle en Uruguay, lorsque le photographe a découvert, par hasard, une grotte dans laquelle il y avait des peintures rupestres. Il s'est alors questionné sur le rôle de ces œuvres issues de la préhistoire. Il établit un parallèle entre la peinture rupestre dans les grottes et le travail du photographe dans les chambres noires. Il replonge ainsi dans nos origines, au cœur de cette époque où des éléments, devenus fondamentaux dans nos sociétés actuelles, sont nés : le feu, l'art ou encore l'activité de représentation de notre environnement. 


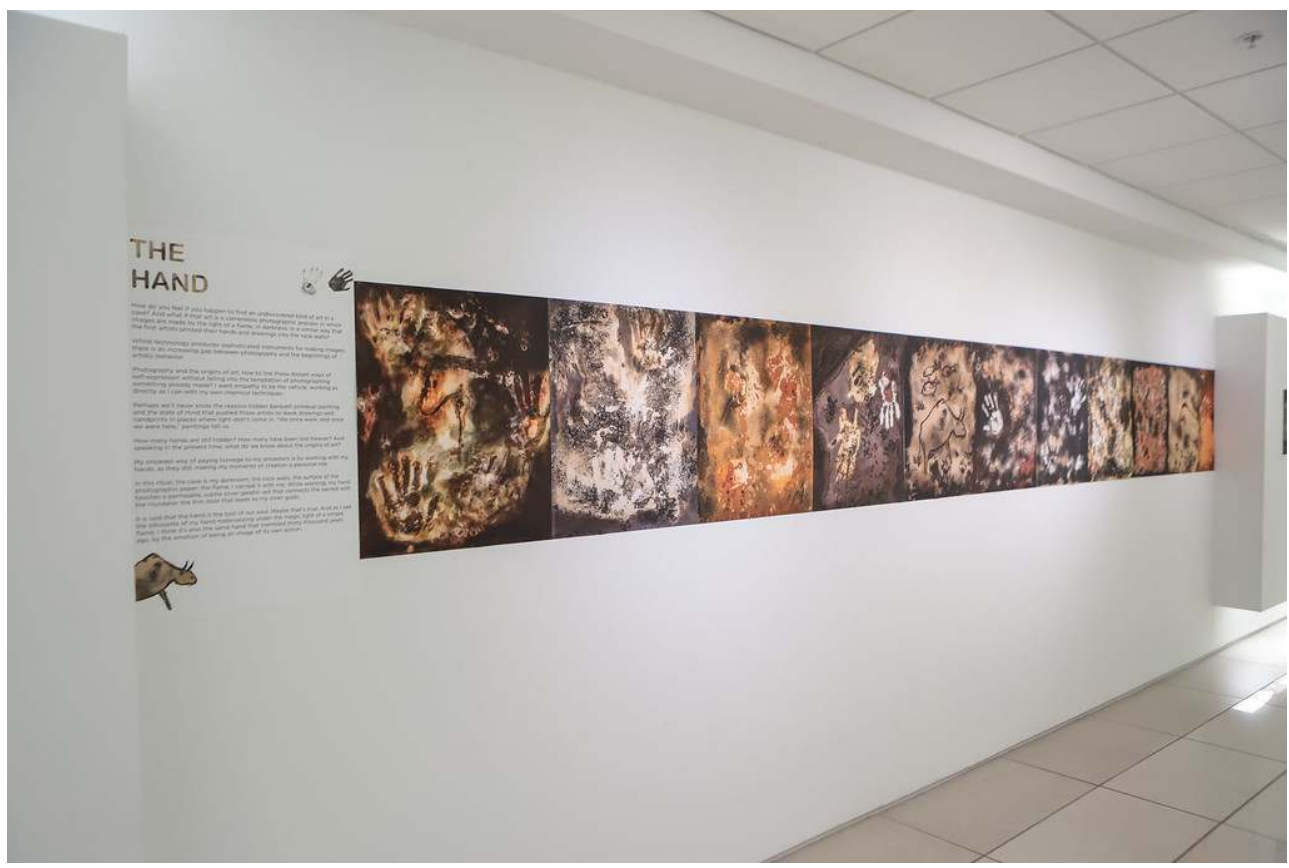

(c) Rafa Cruz, 2018 (reproduit avec l'aimable autorisation du photographe).

L.c. : Roberto Huarcaya et Roberto Fernández Ibáñez utilisent tous deux des techniques à la fois originales et anciennes, afin de représenter des éléments de la nature. À l'ère du numérique, quelle est selon vous l'importance d'utiliser de telles techniques qui, rappelonsle, se passent totalement d'appareils photo?

F.G.B. : Il est vrai que le monde du numérique a connu des évolutions incroyables ces dernières années et, évidemment, les photographes utilisent leur téléphone portable afin de prendre certaines photos. Mais ils cherchent aussi des techniques alternatives; ils explorent, ils testent et ils participent même de la conservation de techniques anciennes. En fait, chaque photographie est une œuvre d'art et suppose, par conséquent, une technique particulière. Par exemple, dans le cas de Roberto Huarcaya, la question était la suivante : comment photographier l'Amazonie, cette forêt immense composée de millions d'arbres dont les feuillages sont si denses? Avec un rouleau de papier photosensible mesurant 30 mètres de long, il répond poétiquement à cette question. Aujourd'hui, toutes les techniques continuent d'être utilisées selon l'intention du photographe, de l'artiste. Roberto Huarcaya et Roberto Fernández Ibáñez n'utilisent pas les techniques numériques, mais celles de la chimie photographique, en explorant les réactions du papier photosensible aux différents produits chimiques utilisés.

L.c. : Les photographies mainstream, prises par des appareils photo et que l'on retrouve un peu partout sur les réseaux sociaux ou dans les journaux, paraissent a priori plus faciles à comprendre, car elles offrent souvent un message explicite. Selon vous, des techniques alternatives, telles que le photogramme ou le cyanotype, risquent-elles de «brouiller» le message ou, au contraire, le rendent-elles plus percutant?

F.G.B: Je crois que les différentes techniques sont complémentaires: les gens comprennent la photographie de Roberto Huarcaya et cela n'est pas incompatible avec des modes de photographie plus mainstream. Toutefois, Roberto Huarcaya et Roberto Fernández Ibáñez font le choix de valoriser la technique plutôt que le résultat final. L'intention ne se retrouve pas seulement dans l'image obtenue : ils cherchent aussi à 
proposer une réflexion sur le processus technique, sur le moyen lui-même, plutôt que sur le produit final. Dans le passé, certaines photos n'étaient pas utilisées car des défauts étaient visibles, comme des poussières ou des zones d'ombre. Or de nombreux photographes contemporains cherchent à incorporer ces « erreurs ", qui sont en réalité produites par la technique elle-même. Cela permet de donner au spectateur une idée de ce qui se passe derrière la représentation et des intentions du photographe. La partie conceptuelle et la partie artistique sont extrêmement importantes. C'est justement le choix de la technique utilisée qui rend la photo unique. Lorsque l'on prend des photos avec notre téléphone, il est facile de les reproduire ou de les copier. Or les photographes contemporains cherchent à produire un objet d'art unique et cela passe par la technique et le processus de production. D'ailleurs, c'est vraiment ce que nous cherchons à valoriser avec l'AMA.

\section{L.c. : D'autres expositions photographiques sont-elles prévues pour les prochains mois ?}

F.G.B. : Oui, la programmation change très régulièrement. Dans la Photogalerie, nous accueillerons bientôt une exposition intitulée On the Move qui s'intéresse aux mouvements humains constants. Ce sont trois artistes venant du Paraguay, du Brésil et du Mexique, qui ont travaillé ensemble sur cette thématique.

Le premier d'entre eux, Juana Barreto Yampey, a cherché à photographier, en noir et blanc, les mouvements dans une gare au Paraguay. De nombreux bus sortent de cette gare routière, pour aller à Buenos Aires, Porto Alegre ou d'autres villes de la région. Il s'intéresse en fait à la question des migrations internes en Amérique du Sud, notamment entre le Chili, le Brésil et le Paraguay. En photographiant ces va-et-vient humains, ses photos retracent le phénomène de dispersion des communautés paraguayennes dans toutes ces régions ainsi que l'évolution des liens avec les proches au travers des distances géographiques.

La seconde photographe, Helena Giestas, travaille sur la question des déplacements internes et, plus précisément, l'exode rural. Sa technique est originale puisqu'elle prend des photos depuis une voiture - soit en étant elle-même en mouvement - de personnes qui sont également en mouvement vers les villes. Elle développe ensuite ses photos, découpe les silhouettes des personnes photographiées et les place à la verticale en donnant seulement à voir le verso de la photo, c'est-à-dire la partie blanche du papier. Autrement dit, il ne reste de la photo prise qu'une multitude de silhouettes blanches positionnées verticalement et superposées. Cela lui permet à la fois d'extraire l'identité individuelle des sujets photographiés, tout en effaçant les informations sur le lieu et le contexte. Elle souhaite ainsi parler du déplacement interne comme un phénomène intemporel, universel et présent dans toutes les sociétés humaines.

Enfin, la dernière photographe, Olivia Vivanco, s'est rendue sur les routes que prennent les migrants pour aller aux États-Unis ainsi que dans les refuges, au Mexique, où ils s'arrêtent pour dormir. Elle photographie les objets que les individus ont choisi d'emmener avec eux: la photo de leur mère ou de leurs enfants, des documents personnels, des roses ou encore des jouets. L'idée centrale de ce projet collectif est de faire prendre conscience aux spectateurs, aux visiteurs, que les déplacements internes ou externes sont universels et intemporels : les gens vont continuer à se déplacer, migrer et bouger.

L.c. : Au final, les photographes dont vous exposez les œuvres ont tous en commun cette volonté d'attirer l'attention sur des enjeux contemporains, tels que le changement climatique, l'exclusion géographique et sociale ou encore les migrations. Selon vous, quelle 
influence ces messages peuvent-ils avoir sur les personnes qui viennent visiter l'AMA et la Photogalerie?

F.G.B.: L'idée n'est pas d'effrayer les spectateurs en leur montrant les problèmes auxquels le monde contemporain fait face, via des photographies choquantes. Au travers d'expositions accessibles aux familles et gratuites, nous souhaitons plutôt raconter une histoire. L'objectif est de créer un espace dans lequel les visiteurs peuvent venir, regarder et apprécier les photos, puis rentrer chez eux et ouvrir une discussion sur des enjeux importants et qui traversent les sociétés de notre continent. Nous travaillons principalement avec deux types de public: d'une part, des touristes qui viennent à Washington pour visiter la ville; d'autre part, un public très spécifique, particulièrement intéressé par la photographie et avec des attentes précises. Ces deux publics ont toutefois en commun cette volonté de se connecter avec ce qu'il se passe dans les Amériques - les modes de vie, les enjeux et problèmes actuels, etc. C'est aussi pour cela que, lorsque nous choisissons les expositions, il y a un enjeu énorme de représentation des différentes sociétés des Amériques. Par exemple, en novembre 2019, je vais aller au Festival GuatePhoto, à Guatemala City. En me rendant dans des festivals sur tout le continent, je m'assure ainsi que toutes les sociétés soient représentées.

L.c. : Finalement, I'AMA semble être un «point de rencontre » entre les réalités et les représentations de toutes les sociétés des Amériques...

F.G.B.: Oui, bien sûr! Et cela est simplement dû au fait que nous partageons une histoire commune, marquée par la colonisation et l'arrivée de courants politiques et culturels, tels que le néo-classicisme, le fascisme, le communisme ou encore le néolibéralisme. De la même manière que nous avons un passé en commun, notre présent est aussi lié. D'où l'importance d'une coopération politique, sociale et culturelle entre les différents pays d'Amérique ; c'est d'ailleurs ce que l'OÉA essaie de promouvoir ! Avec ces expositions, nous essayons aussi de créer de l'empathie entre les différents habitants d'un même continent, entre des individus venus de sociétés différentes mais dont le passé et le présent sont liés.

L.c. : Durant notre première rencontre, vous me disiez qu'il pouvait parfois être difficile pour les photographes de faire leur travail, du fait de pressions de la part des États, si bien que certains d'entre eux devaient protéger ou cacher leurs photos. Pensez-vous que les photographes assument un certain rôle de contre-pouvoir dans les démocraties ?

F.G.B. : En fait, les photographes et les artistes se rendent souvent dans des endroits dans lesquels, traditionnellement, personne ne veut aller. Ils s'intéressent aux marges de la société, qu'elles soient géographiques, sociales ou économiques. Et, dans un objectif de représentation et de justice, l'AMA cherche à promouvoir de tels travaux, qui se rapprochent d'ailleurs du photojournalisme. Le photographe, en tant qu'artiste, a une intention politique. Nous sommes un musée d'art contemporain et il est important de garder cette perspective en tête : l'art est une manière de donner à voir certaines réalités et l'art est nécessairement politique.

Concernant le cas de Roberto Huarcaya, il a dû révéler ses photos aux autorités car il n'avait pas le choix. Il ne pouvait pas voyager avec un énorme sac noir devant la police, qui l'aurait surement pris pour un trafiquant. Il a ainsi dû révéler le contenu de ses photos pour pouvoir les faire sortir de la forêt, un peu comme dans les aéroports...

L.c. : Ceci m'amène à la dernière question de cet entretien. Dans les Amériques, le contexte politique et social contemporain est particulièrement tendu, marqué par de nombreuses 
violations des droits humains. Face à cela, pensez-vous que l'art et la photographie engagée aient un rôle particulier à jouer?

F.G.B. : L'art a eu, a et aura toujours un rôle à jouer par rapport à la vie politique. C'est une certitude. Toutefois, j'aime à analyser le rôle de l'art selon une dichotomie entre action et réaction. Ce qu'il se passe au Brésil, aux États-Unis ou en Argentine par exemple ne sont pas des phénomènes isolés, ce sont des réactions à quelque chose. Aux États-Unis, après avoir élu le premier président afro-américain, Barack Obama, nous observons une réaction au travers de l'élection de l'actuel président. En Amérique latine, on remarque que les dirigeants politiques ont tendance à vouloir conserver le pouvoir. Bref, tout ce qu'il se passe actuellement dans le monde politique c'est le naturel et non pas 1 'exceptionnel. Dans ces va-et-vient politiques constants marqués par des réactions, le photographe cherche à montrer l'exceptionnel. Selon moi, c'est la fonction même de l'art : transformer un objet commun en une chose spéciale et exceptionnelle. C'est là un pouvoir de transformation que nous avons, en tant qu'artistes. Il s'agit ainsi de représenter, montrer, créer et dénoncer pour questionner le naturel.

L.c.: La fonction de l'artiste serait donc de soulever des enjeux et de donner à voir certaines réalités. Selon vous, s'agit-il du rôle des individus et des sociétés d'agir afin de faire changer, de manière concrète et effective, les situations ordinaires ?

F.G.B. : En fait, la question qu'il faut d'abord se poser est la suivante : quel est le rôle de l'art ? Comme je le disais, il s'agit de représenter ce qu'il est en train de se passer afin de provoquer une prise de conscience. Cela peut d'ailleurs se faire de façon plus ou moins subversive. Les individus, lorsqu'ils voient ces photos, peuvent ensuite se questionner, discuter et agir. Au final, en donnant à voir l'exceptionnel, les photographes veulent montrer une chose essentielle, à savoir que l'on peut sortir de la marche naturelle de l'Homme, celle de l'autodestruction, et changer le cours des choses. L'injustice n'est pas irréversible. Le Musée, en mettant en avant l'exceptionnel, est là pour montrer qu'il y a une lueur d'espoir et que chacun peut agir. Par exemple, Roberto Huarcaya n'est pas seulement photographe, mais il est aussi directeur du Centre de l'Image de Lima et il enseigne la photographie à des étudiants en Master. Il agit !

Il y a des lieux pour dénoncer, telle la Commission interaméricaine des droits de l'homme, mais la photographie est plutôt là pour démarrer une conversation qui peut mener à l'action. Car il faut être actif, réagir et trouver des solutions sur le long terme. Je dis cela dans un contexte où, avec l'explosion des réseaux sociaux, on a vu naître une pluralité de réactions virtuelles, souvent dans des espaces numériques qui sont des entre-soi, avec une information et des représentations qui sont fréquemment les mêmes. Avec l'AMA, nous essayons d'être vraiment actifs, en choisissant des photographes qui se questionnent sur les migrations ou encore le développement durable. Il s'agit de ne pas rester paralysés face à une situation : il y a, je crois, une relation dynamique entre le photographe et son œuvre, entre le photographe et le spectateur, entre le spectateur et son environnement social et politique...

L.c. : Merci beaucoup, Fabian, d'avoir partagé votre vision du rôle de la photographie dans notre monde contemporain. Ce fut fascinant de découvrir votre parcours, les expositions que vous avez organisées et votre conception de l'art.

Entretien réalisé au Musée d'art des Amériques, à Washington, D.C., en janvier 2019. 


\section{NOTES}

1. Le kintsugi est une technique japonaise consistant à réparer des sculptures, porcelaines ou céramiques grâce à de la laque sur laquelle est saupoudrée de la poussière d'or.

\section{AUTEUR}

\section{LAURA CAHIER}

Doctorante en Droit Public à l'Université d'Aix-Marseille (UMR 7318 CERIC - ED67), chercheuse invitée au Center for Latin American Studies (CLAS) de l'Université de Georgetown et représentante de l'Institut des Amériques à Washington, D.C. Diplômée d'un Master en Affaires Internationales (Sciences Po Lyon) et d'un Master 2 en Droits de l'homme et Droit humanitaire (Paris 2 Panthéon-Assas). laura.cahier@gmail.com 